\title{
Intertemporal Choice: Decision Making and Time in Software Engineering
}

\author{
Christoph Becker, Dawn Walker, Curtis McCord \\ Faculty of Information \\ University of Toronto \\ Toronto, Canada \\ christoph.becker@utoronto.ca,dawn.walker@mail.utoronto.ca,curtis.mccord@mail.utoronto.ca
}

\begin{abstract}
When making choices in software projects, engineers and other stakeholders engage in decision making that involves uncertain future outcomes. The concept of 'intertemporal choice' describes choices between outcomes at different times in the future. Short-sighted decisions with far-reaching effects are a long-standing cause of concern in the software profession.

Common models to support decisions in software projects use concepts such as expected utility and discount factors to quantify future value and enable trade-off decisions. However, a growing body of behavioral research shows that these normative models do not adequately describe how people actually make choices.

Our objective is to understand how developers and stakeholders actually take trade-off decisions during software projects that involve current and future benefits, and to identify the human and cooperative factors that influence them. This requires empirical research on decision making in SE with a focus on trade-offs across time. To support such research, this paper reports on a systematic literature review that aimed to identify whether the intersection of these concepts has been acknowledged and addressed. We discuss the assumptions about decision makers that underpin existing research and analyze how the role of time has been characterized in the study of decision making in SE.

Based on this review, the paper begins to develop principles for a descriptive framework to characterize intertemporal choices in empirical and behavioral software engineering research.

Index Terms-Behavioral Software Engineering, Intertemporal Choice, Technical Debt, Sustainability Debt, Trade-off decisions, Decision Theory, Sustainability, Systematic Literature Review
\end{abstract}

\section{INTRODUCTION}

When making choices in software development projects, engineers and other stakeholders engage in decision making procedures that involve uncertain future outcomes. These decisions take place in dynamic and changing environments and have often far-reaching effects. The dominant theory of how people make such decisions is the theory of expected utility [1]. Correspondingly, models that aid teams in evaluating options to identify optimal trade-offs typically involve economic considerations such as discount factors to establish the net present value of each option in order to enable a comparison of options in the interest of maximizing utility. In theory, this enables perfectly adequate decisions as long as the estimates are sufficiently accurate.

However, individuals and groups often don't behave as suggested by normative theory. For example, standard frameworks and process models do not explain well how engineers actually make decisions in systems development teams [2], [3]. Many decision support models prescribe ways to evaluate design options through the weighted assessment and comparison of alternatives against stated criteria, but successful expert decision makers often do none of these things when they make important decisions [4]. Similarly, managers often do not apply decision analysis theories even if they are trained to do so [5].

In software engineering (SE) practice, concepts such as 'technical debt' emphasize a continued concern over shortterm-ism in decision making. Technical debt indicates expedient decisions with long-term costs and interest [6], [7], [8]. It corresponds to concerns over the lack of long-term thinking [9], early considerations of long-living effects across the lifecycle [10], and the resulting sustainability debt of software systems [11].

At its core, sustainability is the capacity to endure. It is often defined within the domain of "sustainable development", which "meets the needs of the present generation without compromising the ability of future generations to meet their own needs" [12]. A recent literature review showed that even in the explicit discussion of sustainability requirements - requirements that address the various aspects of technical, social, economic and natural systems to endure - the dimension of time is generally ignored [13].

While we know that normative models do not describe the real decisions that often incur excessive future debt, we don't know how to explain what happens in practice well enough to develop better decision making methods. How do short-term interests and longer-term perspectives interact in such decisions? And if it is true that "there is much to be gained from farsighted thinking that also enables short-term achievements" [9], how can such thinking be supported in practice?

Behavioral research aims to better understand such cognitive and cooperative aspects of human activities, and the recent emergence of behavioral SE [2], [3] suggests a wealth of research questions at the intersection of these fields. Following organizational psychology, these can be grouped according to individual, group and organizational issues [3].

In order to articulate and describe what happens in group decision making in software projects when uncertain future outcomes are weighed against uncertain short-term outcomes, we need more adequate conceptual models for characterizing real-world group decision making activities in SE contexts. 
As an initial step towards such a framework, this discussion paper identifies concepts and frameworks that can shed light on what happens during these team decision making processes.

We first establish key perspectives and terms from decision research in relation to our interest in SE decision making (Section II). Following this, we summarize findings from a systematic literature review we conducted that focused on identifying empirical research on decision making and tradeoffs involving a time factor within the domain of SE (Section III). We then suggest key steps towards developing the descriptive frameworks we seek in Section IV.

\section{DeCISION THEORIES}

\section{A. Normative vs. descriptive theories}

An important distinction is made between normative and descriptive decision theories. Normative theories focus on the identification of the best decision, and model an ideal decision maker. Normative models of how decisions are made in SE commonly assume a rational agent (with reasonable cognitive boundaries) choosing between a set of options according to a value function.

The theory of expected utility stems from game theory [14] and was developed from principles, not empirical study. In contrast, descriptive theories aim to characterize the behavior of actual decision making. As Tversky and Kahneman write, "The modern theory of decision making under risk emerged from a logical analysis of games of chance rather than from a psychological analysis of risk and value. The theory was conceived as a normative model of an idealized decision maker, not as a description of the behavior of real people... the logic of choice does not provide an adequate foundation for a descriptive theory of decision making" [15].

A variety of perspectives have voiced critique of the prevailing normative decision theories, and various alternative conceptions have been proposed. For example, well-known experiments have shown that people do not discount the future linearly [16] and that risk aversion is higher for gains than for losses [15]. More substantively, Tversky and Kahneman showed that some of the foundational axioms of normative decision theory, and in particular expected utility theory, are inconsistent with observable behavior. More radically, Klein's study of expert decision making showed convincingly that experienced decision makers do not actually weigh a set of alternatives against criteria to maximize expected utility when making critical choices [4].

\section{B. Rationalistic vs. naturalistic theories}

The mounting inconsistencies of theory with actual behavior met with different reactions, as described by Beach [17]: Some rejected the behavior as false and 'irrational' and suggested that better training would help overcome these perceived deficiencies. Some adapted the theory while remaining true to the spirit of rationalistic ideals and the metaphor of choice as basis for decision making. This was coupled with a formalist approach centered on tightly controlled laboratory settings used to vary parameters following the scientific method.
Others looked for alternative ways to describe what actually happens in real-world decision making. The latter stream of research came to be described as 'naturalistic' decision research (NDM) [18] and focused on realistic decisions made outside of laboratory settings. Well-known frameworks that describe naturalistic decision making include Recognition Primed Decisions (RPD) and Image Theory [19]. For example, RPD, developed from Klein's studies of experts, suggests that they identify one plausible course of action based on matching the identified patterns to their experience, then rapidly evaluate this course of action and, if needed, consider further options in sequence until a plausible match is found.

A significant shift in perspective within NDM research was the move away from treating deviations from the normative ideal of rational choice as biases that have to be overcome, and instead focus on how to best support decision makers in exercising their knowledge and skills [18].

\section{Decision theories in SE}

Decision making frameworks that aim to support teams in effectively making choices throughout the software development lifecycle often contain assumptions of how people make decisions that remain very close to rational choice. For example, many methods use concepts such as expected utility and net present value based on time-invariant discount rates to represent the economic value of engineering decisions to multiple stakeholders, and focus on making multiple options comparable and measurable through common value functions and criteria weighting. From choosing a development methodology to evaluating release planning, prioritizing requirements and choosing between architectural design options, the assumption is that a team of competent engineers evaluates the options to the best of their knowledge and chooses the option with the highest expected value.

Most commonly, this value is expressed in economic terms, and the incommensurability of multiple criteria is often addressed through expressing utility functions [20]. The Value Based Software Engineering framework aims to base SE decisions more explicitly on a more nuanced understanding of value [21] including perspectives based on Image Theory [22]. Alternative perspectives based on descriptive decision theories have been proposed but remain isolated [23]. While some of course have discussed questions such as power dynamics and politics [24], much of the discussion in theory and practice focuses on how to best estimate that value.

However, a considerable body of evidence suggests the fundamental assumptions of decision making at the basis of such normative models are inadequate descriptions of actual behavior [18], [25]. Empirical research in SE confirms that people don't behave according to these models, even if they are taught to [26]. As da Cunha et al. point out in their empirical study of software project management decisions, whether "simple or complex, the decisions made ... were based on their tacit knowledge, rather than analytical models of decision-making" [26]. 
It is this divergence between prevalent normative models and observed behavior that initially motivated our work. The aim of this work corresponds to the recent emergence of Behavioral Software Engineering, a field aiming to draw in behavioural frameworks and concepts for a better understanding of SE [2], [3], [27].

\section{Trade-offs in Time}

In $\mathrm{SE}$, choices that are expedient in the short-term but create unwanted longer-term consequences have been conceptualized most prominently as 'technical debt', which focuses on engineering choices that create hidden costs [7]: "a design or construction approach that's expedient in the short term but that creates a technical context in which the same work will cost more to do later than it would cost to do now (including increased cost over time)" [28].

The debt metaphor aims to make these hidden costs visible and manageable. In a much broader perspective, the notion of sustainability debt expands the metaphor to direct and indirect effects across all dimensions of sustainability [11].

Research in psychology and behavioral economics has termed choices that involve trade-offs across time "intertemporal choice" and defined them as "decisions involving tradeoffs among costs and benefits occurring at different times" [16]. Researchers have developed a number of theories of such choices [29] and demonstrated that straightforward assumptions about how decision makers evaluate and discount the future are often misguided and wrong [16].

Frederick provides a comprehensive overview of future discounting [16]: Discounted Utility (DU) is commonly used as a normative standard for cost-benefit analysis and policy making in economics to model the value of future events. DU is similar to Net Present Value, as it applies a discounting factor for future outcomes. However, it has been shown to be an incorrect simplification that reduces what economists call "intertemporal choice" to a discount rate that is known to be inconsistent with many actual behaviors. The authors classify a number of anomalies that cannot be explained by DU, such as observed preferences across time that are inconsistent with the linearity of discounting.

Based on a thorough review of prior research, Frederick et al. identify "spectacular disagreement among dozens of studies" and a wide-spread presence of very high implicit discount rates. They point out that similar to other normative decision theories, DU was "never empirically validated as the appropriate model for intertemporal choice". They conclude that linear discount factors are too simplistic and hyperbolic discount functions are more realistic, and that uncertainty plays a role in intertemporal preferences. Finally, many reasons for time preferences in people lie not in the time in which the events are scheduled to occur but by the perceived utility of the occurrence, and studies show that such choices "may be dramatically influenced by people's level of understanding of how their preferences change" [16].

Weber discusses the role of perception in intertemporal choices and the question why long-term, long-distance risks are not moving us to act, with a particular focus on global warming. Her findings suggest that 'the affective system ... has much greater influence over decisions under risk and uncertainty ... than the analytical processing system." She highlights the role of group factors and culture as well as affect: "Abstract representations of consequences in the distant future lack the concrete associations that are connected to emotional reactions" while "concrete representations of choice alternatives in the present or the immediate future tend to be saturated with affective associations" [30].

These selected findings illustrate the need to investigate more specifically how these factors affect decision making involving intertemporal choice in engineering teams.

\section{A Systematic Literature ReVieW}

\section{A. Objective}

To establish better clarity on current views in SE, we conducted a Systematic Literature Review (SLR). As a key step to developing a descriptive framework for intertemporal choices in SE, the literature review aimed to identify whether the intersection of these concepts has been acknowledged and addressed; describe which perspectives and assumptions about decision makers underpin existing research; and analyze how the role of time has been characterized in the study of decision making in SE.

Because of our aim to better understand how and why software practitioners incur sustainability debt in practice, we are interested in distinguishing normative models theorizing about decision-making in SE from descriptive, empirical accounts of how trade-off decisions relating to time are made in software design projects. We thus map empirical and other types of research of decision making, then analyze empirical research in detail in order to understand the assumptions of decision making models that underpin this research.

We aim to reveal how trade-off choices have been conceptualized within SE so far, identify gaps in how decision making is reviewed and investigated, and map how SE literature approaches making trade-offs over time.

The detailed SLR protocol is available at [31]. The SLR followed the common protocol [32] with minor deviations. We will here summarize selected findings to the following questions: (RQ1) Which empirical research in SE has studied trade-off decisions involving time? and (RQ2) Which assumptions on decision making underpin these studies?

\section{B. Method}

1) Terminology: As the term 'intertemporal choice' describes precisely our area of interest, we conducted early searches to identify whether there has been explicit attention to this body of literature. The results suggested that no research has explicitly focused on the concepts and theories of intertemporal choice in this field, as illustrated in Figure 1.

2) Search strategy and results: As a consequence, we adopted a broad and inclusive search strategy for time AND "decision making" AND "software engineering" in Scopus, IEEE Explore and the ACM digital library, then applied 


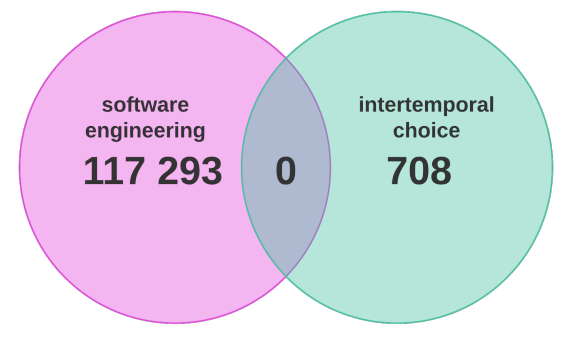

Figure 1: No research was found at the explicit intersection of Intertemporal Choice and Software Engineering.

Table I: Overview of search results for main search.

\begin{tabular}{|l|c|}
\hline Total Number of Search Results & 889 \\
\hline $\begin{array}{l}\text { Total number of results after duplicate removal and } \\
\text { exclusion criteria applied }\end{array}$ & 652 \\
\hline Number selected after preliminary relevancy review & 307 \\
\hline Number selected after voting & 155 \\
\hline
\end{tabular}

standard inclusion and exclusion criteria to identify peerreviewed publications within the domain scope of SE. Table I summarizes search and filter statistics.

\section{Mapping research types}

Our analysis mapped empirical and other research, identified studies focused on trade-off decisions, and analyzed a subset of empirical studies in detail. We distinguished:

1) whether the article discusses a trade-off decision;

2) dimensions of trade-off, including cost, functionality, quality, risk, time, and value; and

3) research methodology, grouped as

a) Empirical methods were used to study decisions.

b) Empirical methods were used, but the object of empirical study was not a decision.

c) The paper is a literature review or mapping study.

d) The research was not empirical, i.e. was theoretical or attempted to develop a model.

We discuss findings to selected research questions below.

D. Which empirical research in SE has studied trade-off decisions involving time?

Figure 2 shows the segments that arise from the analysis and highlights that only a small portion of the identified work investigated specific trade-off decisions empirically. These are discussed in detail further below.

Time is addressed frequently across most types of research: empirical (7), empirical component (19), non-empirical (12), and literature review (0). However, the role of time is of course not always intertemporal. Time surfaces:

- As the object of effort estimation: How much time will each of these options take?

- As a factor in project management: How much time is available to the team?

- As an attribute of decision making: How much time does it take to make a choice?



Figure 2: Segments according to research focus (sum=155).

- As a factor in scheduling, the closest to intertemporal choice: Should we release now or later?

A detailed qualitative analysis of the role of time in the 13 empirical studies revealed that in this set of papers, time is discussed most importantly as

- a constrained resource in software projects,

- the time it takes to apply a method (e.g. designed by the researcher) in a project, and often a measure of that time,

- an axis of discrete units 'of time' over which a sequence of events take place,

- the time to market or the time to delivery, and

- as an axis of change on which to pick suitable moments for action.

It is the last conception where intertemporal decisions arose in particular in two specific forms, each represented by two distinct papers: Technical Debt management raises questions around when to repay, and papers discussed how these decisions are being made. Release planning raises questions of timing and of which requirements to prioritize and include for a given release. However, in neither of these cases explicit attention was given to behavioral insights into how decision makers arrive at their choices.

E. Which assumptions on decision making underpin these studies?

The predominant model of decision making, so dominant that it is normally not made explicit, is a normative model that builds on a Taylorist management perspective focused on efficiency and effectiveness, to be measured in as scientifically accurate a manner as feasible. Decision making assumes the presence and validity of normative theories of decision analysis in which clearly defined options are weighed against stated criteria to determine the best choice. The actual choosing is then often presumed to be unproblematic [1].

Some authors make this explicit. For example, one team writes: "In most problems, to make a decision, a situation is assessed against a set of characteristics or attributes, also called criteria. Decision making based on various criteria is supported by multi-criteria methodologies" [33]. Rational choice is the 
standard model, sometimes with explicit awareness of its limitations, often articulated in the frame of, or consistent with, bounded rationality.

Yet, awareness is also present that "research has proven that humans make trade-off analyses continuously - if not on the basis of objective measurements then on intuition." [34] One author team explicitly proposes to contrast 'rational' decision making with 'naturalistic' frameworks and categorizes frameworks including Simon's Bounded Rationality, Prospect Theory, Image Theory, and others [35], [23]. The latter paper conducts interviews to develop an understanding of two modes of decision making characterized as rational and naturalistic. The findings suggest a distinction between problem structuring and problem solving. The authors conclude that both modes are employed. The nature of the decision making focus determines which mode dominates: Naturalistic decision making was more prominent for decisions that focused on giving structure to problems, while rational modes dominated in decisions that focused on solving problems [23]. In each mode, aspects of the other were present as well. The authors add that "software designers often use satisficing and singular evaluation in trying different approaches to design", where one option is checked for plausibility rather than being evaluated against other options, as described by RPD and other naturalistic models [4].

However, the normative, rational decision making model also dominates the empirical papers that addressed the notion of trade-offs across time more explicitly.

In Martini's study of technical debt management [36], time is prominent: "[technical debt] instantiates a relationship between the cost and the impact of a single sub-optimal solution over time. In particular, the metaphor stresses the short-term gain given by a sub-optimal solution against the long-term one considered optimal". Decisions have to be taken at the right time and have to anticipate uncertain future outcomes. The assumptions that are surfaced about the decision makers suggest that in the presence of perfect information, they would weigh the options using all available criteria in order to take the correct, 'optimal' decision. Their choice is assumed to be rational, contingent upon and bounded by the availability of information.

In Racheva's study of requirements prioritization in an agile environment at inter-iteration time-the moment "when requirements are re-prioritized in the face of project uncertainties" [37]-the trade-offs consist in choosing what to do at the next iteration and what to postpone or discard. Over time, more information will be delivered and less of the limited resource of time will be available to act on it: "The client can wait to the last responsible moment (as it is called in [18]) to make his decision ... The term 'responsible' means that the client needs to understand the last point of time to make a decision without affecting the delivery of the project." The framework that is introduced aims to provide a conceptual frame for uncertainty over time by introducing Real Options Analysis. Given the agile focus, it is unsurprising that the research focus lies on grounding proposals on empirical insights and be responsive to the actual behavior of practitioners. The underpinning assumptions remain implicit, but build on ideas of bounded rationality.

\section{F. Discussion}

Trade-off decisions in SE have been studied and modelled for a long time. However, the role of time in the studies we identified focuses primarily on time as a limited resource and a ticking clock. However, some work, such as technical debt research, has foregrounded the attention to trade-off decisions across time.

A number of studies have suggested that normative models are inadequate in explaining how people actually take decisions. Behavioral perspectives and empirical research are needed to provide new and deeper understanding on the practice of SE [3]. This suggests that more descriptive research is needed to provide a bottom-up empirically grounded description of decision making. We are aware of some studies within the domain of SE, but have identified none yet that focused on time trade-offs.

Some awareness is evident in the empirical literature that normative decision theory has limited relevance for descriptive and explanatory purposes. Yet, little empirical work surfaced that explicitly pursues empirically grounded, descriptive approaches, and none that studied trade-offs in time in depth.

The most explicit discussion of intertemporal choice and trade-offs were found in decisions about technical debt management. This suggests that the body of work on technical debt should be analyzed in more depth to characterize the tension between normative decision theory and descriptive approaches, and to identify opportunities for improving our understanding of trade-off decision making in practice.

\section{RESEARCH DIRECTIONS}

From the above, many questions arise that merit further consideration. We will highlight some that arise from our literature review and are particularly relevant to our interest in sustainability debt.

\section{A. Intertemporal choice in technical debt}

What assumptions underpin existing research on intertemporal choices regarding technical debt management? To prepare the empirical study of time trade-offs, an analysis of the technical debt corpus is the logical next step: This work is very clearly bounded and focused on a closely related area of high relevance for the SE community. As part of the literature review, we have prepared a text corpus with the identified 231 papers that can be analyzed quantitatively [31]. This will make it possible to use complementary techniques such as corpusassisted discourse analysis to identify the associations and meanings attributed to time trade-off decisions in the domain of technical debt management.

\section{B. Goals and time horizons}

What is the role of goal setting and time horizons in intertemporal group decision making? It is clear that the 
framing of decisions plays an important role in judgment and decision making [38], and that in software project environments, explicit and concrete goals play an important role in influencing preferences and behavior [39].

Since the discounting of future outcomes clearly does not function linearly, and differences between outcomes at later point in the future become increasingly less pronounced, the question arises: Which influence do explicit and implicit time horizons have on decision outcomes?

\section{The framing of choices}

The importance of framing for decision making is undisputed. In many project situations, the construction of this frame is central, and some research suggests that decision makers seem to rely heavily on naturalistic approaches in this process [23]. The introduction of metaphors such as technical debt and sustainability debt very intentionally aims to re-frame issues that often remain invisible so that they become part of the decision frame and can be more effectively included [11]. However, the influence of such frames on decision makers' evaluations and choices remains unclear.

\section{The adequacy of decision theories}

Previous studies have aimed to demarcate initial sets of boundary conditions under which naturalistic and rationalistic decision making frameworks seem more adequate. However, these boundaries are far from clear. Under which conditions is the common assumption that engineers will behave more boundedly rational than others justified?

Recent insights from neuroscience provide some perspectives that could reconcile rational and naturalistic perspective by describing decision making as a process in which multiple systems within the brain interact in 'parallel-competitive' or 'default-interventionist' ways [1], [40]. Typically, one is described as emotional, affective, and intuitive, the other as slow, analytic, and deliberative. However, how they interact in given scenarios is far from clear [1].

As Loewenstein highlights, "50 years of dominance of the rational choice model has left so many important questions unanswered" [1], but there has been considerable resistance in economics against questioning established normative models in light of behavioral and neuroscientific evidence [1]. Similar resistance could be expected in engineering.

\section{E. Politics and group dynamics}

Intertemporal group decision making is a relatively recent area of study [41], [42], and it is unclear if currently identified factors can explain the dynamics of project teams well.

With technical debt, those who incur the debt will often be closely related to those who will have to pay the interest. Even in these cases, the lack of continuity between what psychologists call 'the experiencing self' and 'the remembering self' [43] can make it difficult for developers to feel affected. Moreover, broader effects such as sustainability debt are often externalized and may be carried by other stakeholders in the future [11].
Considering the importance of stakeholder dynamics and power relationships for an understanding of what happens in software projects [24], understanding how teams incur debt whose effects may not be incurred on those who take the choices will require a better understanding of psychological distance, affect and politics.

\section{CONCLUSIONS AND OUTLOOK}

Loewenstein describes that behavioral economics "incorporates more psychologically realistic assumptions to increase the explanatory and predictive power of economic theory", and Behavioral SE has similar aims for SE theory [3]. In this article, we discussed the particular subfield of intertemporal choice, one of three areas highlighted by Loewenstein besides 'decision making under risk and uncertainty' and 'social decision making', all of which interact in the processes where teams incur technical and sustainability debt.

To understand these processes, the study of decision making in software engineering needs to be enriched by behavioral insights, neuroeconomics and social science perspectives [44], including studies that consider issues of power and conflict and how these interact with intertemporal choice and the framing of decisions. Only an integration will allow us to better understand these decisions so we can begin to address difficult questions such as the above. An appropriate descriptive framework to address the underlying research questions that motivate our work must, at a minimum:

1) be empirically grounded,

2) incorporate descriptive models of decision making in natural settings,

3) enable studies into how and why stakeholders incur technical debt and, in a broader perspective, sustainability debt,

4) address psychological distance and how the definition of system boundaries and stakeholders influences perception, affect and judgment [30],

$5)$ incorporate power dynamics and stakeholder dependencies [24], and

6) distinguish individual, group and organizational factors in group decision making to support the separation of identifiable factors and the identification of relationships between these levels.

The articulation of a descriptive conceptual framework for studying intertemporal choice in SE should enable empirical studies and a conversation about the individual, group and organizational factors that influence such group decision making processes.

Our own work will aim to characterize intertemporal group decisions in a set of software projects to develop a descriptive account that supports insights into the dynamics of group decision making in this context. The further aim is to develop collaborative Action Research models to support more sustainable design choices in software engineering building on these insights. 


\section{ACKNOWLEDGEMENTS}

Part of this work was supported by NSERC through RGPIN2016-06640, and by the Connaught Fund. Thanks to Ruzanna Chitchyan and Stefanie Betz for reviewing the literature review protocol.

\section{REFERENCES}

[1] G. Loewenstein, S. Rick, and J. D. Cohen, "Neuroeconomics," Аппи. Rev. Psychol., vol. 59, pp. 647-672, 2008.

[2] P. Lenberg, R. Feldt, and L.-G. Wallgren, "Towards a Behavioral Software Engineering," in Proceedings of the 7th International Workshop on Cooperative and Human Aspects of Software Engineering, ser. CHASE 2014. New York, NY, USA: ACM, 2014, pp. 48-55.

[3] P. Lenberg, R. Feldt, and L. G. Wallgren, "Behavioral Software Engineering: A Definition and Systematic Literature Review," Journal of Systems and Software, vol. 107, pp. 15-37, Sep. 2015.

[4] G. A. Klein, Sources of Power: How People Make Decisions. Cambridge: MIT Press, 1998.

[5] D. Isenberg, "How Senior Managers Think," Harvard Business Review, 1984.

[6] W. N. Behutiye, P. Rodriguez, M. Oivo, and A. Tosun, "Analyzing the concept of technical debt in the context of agile software development: A systematic literature review," Information and Software Technology, vol. 82, pp. 139-158, Feb. 2017.

[7] N. A. Ernst, S. Bellomo, I. Ozkaya, R. L. Nord, and I. Gorton, "Measure It? Manage It? Ignore It? Software Practitioners and Technical Debt," in Proceedings of FSE, ser. ESEC/FSE 2015. New York, NY, USA: ACM, 2015, pp. 50-60.

[8] Z. Li, P. Avgeriou, and P. Liang, "A systematic mapping study on technical debt and its management," Journal of Systems and Software, vol. 101, pp. 193-220, Mar. 2015.

[9] P. G. Neumann, "The Foresight Saga, Redux," Commun. ACM, vol. 55, no. 10 , Oct. 2012

[10] C. Becker, "Sustainability and longevity: Two sides of the same quality?" in Proceedings of the 3rd International Workshop on Requirements Engineering for Sustainable Systems (RE4SuSy), vol. 1216. CEUR Workshop Proceedings, 2014.

[11] S. Betz, C. Becker, R. Chitchyan, L. Duboc, S. Easterbrook, B. Penzenstadler, N. Seyff, and C. Venters, "Sustainability Debt: A Metaphor to Support Sustainability Design Decisions," in Proceedings of the 4th International Workshop on Requirements Engineering for Sustainable Systems (RE4SuSy), vol. 1416. Ottawa, ON, CA: CEUR Workshop Proceedings, Aug. 2015, pp. 55-63.

[12] G. H. Brundtland, Report of the World Commission on Environment and Development: Our Common Future. United Nations, 1987.

[13] C. C. Venters, N. Seyff, C. Becker, S. Betz, R. Chitchyan, L. Duboc, D. McIntyre, and B. Penzenstadler, "Characterising sustainability requirements: A new species, red herring, or just an odd fish?" in Proceedings of the 39th International Conference on Software Engineering Companion. ACM, 2017.

[14] J. Von Neumann and O. Morgenstern, Theory of games and economic behavior. Princeton university press, 1944.

[15] A. Tversky and D. Kahneman, "Rational choice and the framing of decisions," Journal of business, pp. S251-S278, 1986.

[16] S. Frederick, G. Loewenstein, and T. O'donoghue, "Time Discounting and Time Preference: A Critical Review," Journal of Economic Literature, pp. 351-401, 2002.

[17] L. R. Beach and R. Lipshitz, "Why classical decision theory is an inappropriate standard for evaluating and aiding most human decision making." in Decision making in action: Models and methods, G. A. Klein, J. Orasanu, R. Calderwood, and C. E. Zsambok, Eds. Ablex Publishing, 1993.

[18] G. A. Klein, Ed., Decision making in action : models and methods. Norwood, N.J.: Ablex Pub., 1993.

[19] R. Lipshitz, "Converging themes in the study of decision making in realistic settings," in Decision making in action: Models and methods, G. A. Klein, J. Orasanu, R. Calderwood, and C. E. Zsambok, Eds. Ablex Publishing, 1993.

[20] R. L. Keeney and H. Raiffa, Decisions with multiple objectives: preferences and value trade-offs. Cambridge university press, 1993.

[21] S. Biffl, A. Aurum, B. Boehm, H. Erdogmus, and P. Grünbacher, Valuebased software engineering. Springer Science \& Business Media, 2006.
[22] M. Berry and A. Aurum, "Measurement and decision making," in ValueBased Software Engineering. Springer, 2006, pp. 155-177.

[23] C. Zannier, M. Chiasson, and F. Maurer, "A model of design decision making based on empirical results of interviews with software designers," Inf. \& Softw. Tech., vol. 49, no. 6, pp. 637-653, Jun. 2007.

[24] A. Milne, "Power and Politics in Requirements Engineering: Embracing the Dark Side?" Requirements Engineering, vol. 17, no. 2, pp. 83-98, Jun. 2012.

[25] R. Lipshitz, G. Klein, J. Orasanu, and E. Salas, "Taking stock of naturalistic decision making," Journal of Behavioral Decision Making, vol. 14, no. 5, pp. 331-352, Dec. 2001.

[26] J. A. O. G. da Cunha, F. Q. B. da Silva, H. P. de Moura, and F. J. S. Vasconcellos, "Decision-making in Software Project Management: A Qualitative Case Study of a Private Organization," in Proceedings of the 9th International Workshop on Cooperative and Human Aspects of Software Engineering, ser. CHASE '16. New York, NY, USA: ACM, 2016, pp. 26-32.

[27] R. Hofman, "Behavioral Economics in Software Quality Engineering," Empirical Software Engineering, vol. 16, no. 2, pp. 278-293, Aug. 2010.

[28] S. McConnell, “Technical Debt," 2007. [Online]. Available: http: //www.construx.com/10x_Software_Development/Technical_Debt/

[29] G. Loewenstein, D. Read, and R. F. Baumeister, Time and Decision: Economic and Psychological Perspectives of Intertemporal Choice. Russell Sage Foundation, Feb. 2003.

[30] E. U. Weber, "Experience-Based and Description-Based Perceptions of Long-Term Risk: Why Global Warming does not Scare us (Yet)," Climatic Change, vol. 77, no. 1-2, pp. 103-120, 2006.

[31] C. Becker, D. Walker, and C. McCord, "A systematic literature review on intertemporal choice in software engineering - protocol and results," arXiv preprint arXiv:1701.08310 [cs.SE], 2017. [Online]. Available: http://arxiv.org/abs/1701.08310

[32] B. Kitchenham, O. P. Brereton, D. Budgen, M. Turner, J. Bailey, and S. Linkman, "Systematic literature reviews in software engineering a systematic literature review," Information and software technology, vol. 51, no. 1, 2009.

[33] M. Simão Filho, P. R. Pinheiro, and A. B. Albuquerque, "Task allocation in distributed software development aided by verbal decision analysis," in Software Engineering Perspectives and Application in Intelligent Systems. Springer, 2016, pp. 127-137.

[34] R. Van Solingen, "Measuring the ROI of software process improvement," IEEE software, vol. 21, no. 3, pp. 32-38, 2004

[35] C. Zannier and F. Maurer, "A qualitative empirical evaluation of design decisions," in ACM SIGSOFT Software Engineering Notes, vol. 30, no. 4. ACM, 2005, pp. 1-7.

[36] A. Martini and J. Bosch, "An empirically developed method to aid decisions on architectural technical debt refactoring: Anacondebt," in Proceedings of the 38th International Conference on Software Engineering Companion. ACM, 2016, pp. 31-40.

[37] Z. Racheva and M. Daneva, "How do real options concepts fit in agile requirements engineering?" in 2010 Eighth ACIS International Conference on Software Engineering Research, Management and Applications. IEEE, 2010, pp. 231-238.

[38] A. Tversky and D. Kahneman, "The Framing of Decisions and the Psychology of Choice," Science, vol. 211, no. 4481, pp. 453-458, 1981.

[39] T. K. Abdel-Hamid, K. Sengupta, and C. Swett, "The impact of goals on software project management: An experimental investigation," MIS quarterly, pp. 531-555, 1999.

[40] J. S. B. Evans, "Dual-processing accounts of reasoning, judgment, and social cognition," Annu. Rev. Psychol., vol. 59, pp. 255-278, 2008.

[41] D. O'Hora, R. Carey, A. Kervick, D. Crowley, and M. Dabrowski, "Decisions in Motion: Decision Dynamics during Intertemporal Choice reflect Subjective Evaluation of Delayed Rewards," Nature Scientific Reports, vol. 6, p. 20740, Feb. 2016

[42] E. Carbone and G. Infante, "Are groups better planners than individuals? An experimental analysis," Journal of Behavioral and Experimental Economics, vol. 57, pp. 112-119, Aug. 2015.

[43] D. Kahneman, Thinking, fast and slow. Macmillan, 2011

[44] Y. Dittrich, R. Klischewski, and C. Floyd, Eds., Social thinking-software practice. Cambridge, Mass.: MIT Press, 2002. 